\title{
ALGUNOS PROBLEMAS DEL AGOTAMIENTO DE LOS DERECHOS DE PROPIEDAD INTELECTUAL RESPECTO DE LAS LICENCIAS DE SOFTWARE*
}

\section{SOME PROBLEMS IN RELATION THE EXHAUSTION OF INTELLECTUAL PROPERTY RIGHTS REGARDING SOFTWARE LICENSES}

\author{
Juan Camilo Contreras-Jaramillo**
}

Fecha de recepción: 24 de marzo de 2016 Fecha de aceptación: 24 de mayo de 2016

Disponible en linea: 31 de mayo de 2016

\section{Para citar este artículo/To cite this article}

\begin{abstract}
Contreras-Jaramillo, Juan Camilo, Algunos problemas del agotamiento de los derechos de propiedad intelectual respecto de las licencias de software, 132 Vniversitas, 111-132 (2016). http://dx.doi.org/10.11144/Javeriana. vj132.apad doi:10.11144/Javeriana.vj132.apad
\end{abstract}

* Artículo de reflexión producto del proyecto de investigación Límites y Restricciones a la Propiedad, Pontificia Universidad Javeriana.

** Profesor del Departamento de Derecho Privado, Facultad de Ciencias Jurídicas, Pontificia Universidad Javeriana. Contacto: juancontreras@javeriana.edu.co 


\section{RESUMEN}

La figura del agotamiento de los derechos de propiedad intelectual en relación con los contratos de licencia de software genera los mismos problemas que se pueden presentar en otros negocios jurídicos que tienen por objeto otras obras protegidas por el derecho de autor. Hoy, por ejemplo, se discute sobre cuál es el acto jurídico que puede habilitar el agotamiento de los derechos, cuáles son los derechos que se agotan mediante esta figura; incluso, respecto de la forma en que los contratos de licencia deben ser interpretados cuando hay diferencias sobre las disposiciones que pueden o no dar origen al agotamiento. Todos estos cuestionamientos son igualmente aplicables a los programas de computador y a las obras musicales o literarias. Sin embargo, el presente artículo, fruto de una ponencia del autor en la Universidad de Chile, trata en concreto dos problemas relevantes para la industria del software y los contratos de licencia allí utilizados; estos son la interpretación de los contratos y la calificación de la naturaleza de las copias objeto de descargas digitales. Los dos problemas identificados son enfrentados a la figura del agotamiento de los derechos de propiedad intelectual para concluir la necesidad de revisar los criterios de interpretación utilizados en estos contratos, y para evidenciar la necesidad de volver a categorizar las denominadas copias digitales como "bienes digitales tangibles".

Palabras clave: propiedad intelectual; agotamiento de los derechos de propiedad intelectual; software; bienes digitales tangibles; programas de computador; UsedSoft 


\section{ABSTRACT}

The first sale doctrine in relation to software license agreements generates the same problems that may arise in other contracts that deals other copyrighted works; today, the doctrine discusses which dispositions enable the exhaustion of rights, what rights are exhausted by this figure, or even how to interpreted licenses agreements. All these questions are equally applicable to software, as they are to musical or literary works. However, this paper, which is the result of a presentation made by the author at the University of Chile, discuss in particular two issues relevant to the software license agreements; these are the interpretation of these contracts and the nature of the digital downloads. The two problems identified here confronted with the first sale doctrine to conclude the must needed review to the interpretation criteria used in these contracts, as well as to demonstrate the need to re-categorize the so-called digital copies, now as "tangible digital goods".

Keywords: Intellectual property; exhaustion of intellectual property rights; first sale; software; digital tangible goods; computer programs; UsedSoft

\section{SUMARIO}

I. Precisiones SemÁnticas Sobre LaS Licencias de SOFTWARE. -II. La Figura DEL AGOTAMIENTO DE LOS DERECHOS DE PROPIEDAD INTELECTUAL Y ALGUNOS DE SUS PRoblemas. - A. La interpretación de los contratos de licencia. B. El objeto sobre el que recae el agotamiento. -1 . Los bienes digitales tangibles. -Conclusiones. -Bibliografía. 


\section{PRECISIONES SEMÁNTICAS SOBRE LAS LICENCIAS DE SOFTWARE}

Vender, ceder, prestar, alquilar o licenciar no son sinónimos legales. No obstante, en la industria de los programas de computador por diferentes motivos suelen ser utilizados como equivalentes. En algunos casos, ciertos factores tributarios o impositivos han incentivado el uso indiscriminado de estos términos ${ }^{1}$; en otros casos, el interés económico o el modelo de negocio han favorecido una cierta apatía hacia la precisión terminológica.

Sobre la diferenciación de estos términos, es conveniente ir de lo general a lo particular. Primero se debe considerar que los derechos de propiedad intelectual son, en uno de sus aspectos, derechos patrimoniales configurados bajo el esquema de relaciones jurídicas de derecho real (es decir, una relación directa entre el titular y el bien protegido). En este sentido, el ejercicio de las facultades derivadas de este derecho obedecerá a la voluntad de su titular, acotada por los límites establecidos en interés general o en interés particular.

El ejercicio de estas facultades por parte del titular puede darse en una mayor o menor graduación: (i) bien sea para transferir (disponer) derechos o facultades, o para (ii) autorizar el aprovechamiento (uso y/o goce) de tales derechos. En otras palabras, el titular de los derechos puede (i) ceder (onerosa o gratuitamente) su posición de titular a favor de otra persona o puede (ii) licenciar el aprovechamiento de algunas de sus facultades de forma limitada a favor de otra persona.

Como resultado de lo anterior, hay una gran diferencia entre una manifestación de voluntad encaminada a ceder derechos y otra encaminada a licenciarlos; básicamente, ceder corresponde a una disposición de derechos para transferirlos de un patrimonio a otro (el tercero será en adelante el nuevo titular) y la licencia expresará la intención de autorizar de forma limitada el uso o el goce de derechos que el titular "presta" a un tercero; de esta forma, este último no

1 Aunque en el régimen tributario colombiano sí diferencia entre venta y licencia (Concepto 063288 de la DIAN), los efectos en las declaraciones y pagos del impuesto sobre las ventas ha llevado a que varios contratos de licencia sean denominados "venta de software" y viceversa. Colombia, Dirección de Impuestos y Aduanas Nacionales, DIAN, Concepto 063288, 16 de agosto de 2007. Disponible en: http://www.notinet.com.co/pedidos/conc-63288-07.doc 
dejará de ser un simple autorizado (una relación muy similar a la de un propietario y su arrendatario).

Así, se puede afirmar que una licencia es un acto jurídico (contrato o manifestación unilateral de la voluntad), mediante el cual el titular de un derecho de propiedad intelectual permite que un tercero use o goce la obra protegida, de forma limitada y sin perder la condición de titular de ese bien inmaterial.

Una diferencia adicional que se debe presentar acá, debido a la realidad de los contratos de licencia de software, es aquella que está sustentada en la teoría del corpus mysticum y el corpus mechanicum $^{2}$. Bajo esta explicación filosófica se diferencia entre las relaciones jurídicas que pueden consolidarse sobre un bien inmaterial y las que lo harán respecto de uno material; es decir, se distingue entre la "propiedad intelectual" sobre el primero y la "propiedad ordinaria" sobre el segundo, aun cuando un mismo bien material puede incorporar también bienes inmateriales.

Ahora, para el caso de las licencias de software, y habiendo dicho que mediante el contrato de licencia no hay una transferencia de derechos de propiedad intelectual y que el titular de estos derechos mantiene tal condición, se puede añadir que el acto de autorizar el uso y/o el goce del software se realiza mediante la entrega de un soporte material que contiene una copia del bien inmaterial; sin esa entrega resultaría imposible que el licenciatario pudiese realizar los actos autorizados por medio de la licencia, por lo menos en la mayoría de las modalidades de licenciamiento, exceptuando, por ejemplo, aquellas que están más cercanas a la prestación de un servicio, como las licencias SaaS (Software as a Service).

Aun cuando la entrega del soporte material en el contrato de licencia constituye un elemento de la esencia ${ }^{3}$ de este tipo de contratos, este acto (la entrega del soporte material) normalmente ostenta una relevancia económica de menor envergadura en el modelo de negocio detrás de la licencia. Es decir, tanto licenciante como licenciatario centran toda su "atención contractual" en la licencia del bien inmaterial (corpus mysticum), mientras que desatienden por

2 Hermenegildo Baylos-Corroza, Tratado de derecho industrial, 136 ( $3^{\text {a }}$ ed., Civitas, Navarra, 2009).

3 Sin este elemento el contrato derivaría en otro contrato diferente. Colombia, Ley 57 de 1887, Código Civil, artículo 1502, requisitos para obligarse. Disponible en: http://www.secretaria senado.gov.co/senado/basedoc/codigo_civil.html 
completo la relación contractual sobre el soporte material (corpus mechanicum). Tal situación se presenta, debido a que las partes suelen reconocer un valor de mercado casi irrelevante al soporte material, de forma que la suerte de este bien no merece una regulación contractual (al parecer de las partes); entonces, las licencias de programas de computador suelen concentrarse en el fondo (la licencia sobre los derechos de uso y goce) y no en la forma (condiciones bajo las cuales se entrega la copia del software).

Como resultado de lo anterior, las licencias de uso de software son contratos entre dos partes, una de las cuales se obliga para con la otra a permitirle el uso de un software, sin transmitir derechos de propiedad intelectual, mediante la entrega de una copia incorporada a un archivo, CD, memoria u otro tipo de soporte. Sin embargo, este contrato, por lo general, no determina las condiciones en que se entrega este soporte. Y aunque este pueda ser menospreciado en este momento contractual, posteriormente adquiere un valor relevante en los mercados secundarios o incluso en las operaciones de reestructuración empresarial, y la desregulación contractual sobre estos temas generará graves problemas jurídicos a las dos partes, en especial en relación con el agotamiento de los derechos de propiedad intelectual.

\section{LA FIGURA DEL AGOTAMIENTO DE LOS DERECHOS DE PROPIEDAD INTELECTUAL Y ALGUNOS DE SUS PROBLEMAS}

Aunque determinar la naturaleza de la figura del agotamiento de los derechos de propiedad intelectual es una tarea compleja en la que confluyen diferentes elementos como la propiedad y sus limitaciones, el impacto de la distribución de bienes en el mercado ${ }^{4} \mathrm{o}$ el efecto de los contratos por adhesión ${ }^{5}$; comúnmente, esta figura

4 Ariel Katz, What Antitrust Law Can (and Cannot) Teach about the First Sale Doctrine, 1-58, 6 (University of Toronto, Faculty of Law, Working paper, January 23, 2012). Disponible en: http://ssrn.com/abstract=1845842. GuIDo WestKAMP, Intellectual Property, Competition Rules and the Emerging Internal Market: Some Thoughts on the European Exhaustion Doctrine, 11 Marquette Intellectual Property Law Review, 2, 291-335 (2007). Disponible en: http://scholarship. law.marquette.edu/cgi/viewcontent.cgi?article $=1096 \&$ context $=\mathrm{iplr}$

5 Juan Camilo Contreras-Jaramillo, Origen y sustento del agotamiento de los derechos de propiedad intelectual, 131 Vniversitas, 277-322 (2015). Disponible en: http://revistas.javeriana. edu.co/index.php/vnijuri/article/view/15030/12399 
se cataloga como un límite al derecho de propiedad intelectual ${ }^{6}$, específicamente respecto de la facultad de distribución.

Entendido desde el esquema tradicional de la protección que brinda el derecho de autor ${ }^{7}$, mediante la figura del agotamiento se obtiene como efecto la extinción del derecho de distribución a partir de la primera venta o transferencia de propiedad del original o copias tangibles de la obra ${ }^{8}$. No obstante la aparente simplicidad de la anterior descripción, el desarrollo de cada uno de sus componentes es mucho más profundo9. Hoy, por ejemplo, se discute sobre cuál es el acto jurídico que puede habilitar el agotamiento de los derechos, cuáles son los derechos que se agotan mediante esta figura, incluso, respecto de la forma en que los contratos de licencia deben ser interpretados cuando hay diferencias respecto de las disposiciones que pueden o no dar origen al agotamiento ${ }^{10}$. Desde las discusiones más cercanas a un modelo de negocio completamente analógico, en el que se debate por ejemplo, si la entrega de materiales promocionales

6 Catalogado como limite por el Copyright Act of 1976, en Estados Unidos y la Ley 17.336, en Chile. Estados Unidos, Copyright Act of 1976, 17 United States Code § 109. Disponible en: http://www.copyright.gov/title17/92chap1.html. Chile, Ley 17.336 sobre la Propiedad Intelectual (modificada por la Ley 20.435, 4 de mayo de 2010), 28 de agosto de 1970, en vigor desde el 2 de octubre de 1970. Disponible en: http://www.wipo.int/wipolex/es/details.jsp?id=797

7 Juan Camilo Contreras-Jaramillo, El aparato teórico en la estructura tradicional del sistema de propiedad intelectual (Pontificia Universidad Javeriana, Working paper, enero de 2016). Disponible en: https://www.academia.edu/25275568/WORKING_PAPER_El_Aparato_ $\mathrm{Te} \% \mathrm{C} 3 \% \mathrm{~B} 3 \mathrm{rico}$ en_la_Estructura_Tradicional_del_Sistema_de_Propiedad_Intelectual

8 "Artículo 6. Derecho de distribución. 1. Los autores de obras literarias y artísticas gozarán del derecho exclusivo de autorizar la puesta a disposición del público del original y de los ejemplares de sus obras mediante venta u otra transferencia de propiedad. 2. Nada en el presente Tratado afectará la facultad de las Partes Contratantes de determinar las condiciones, si las hubiera, en las que se aplicará el agotamiento del derecho del párrafo 1 después de la primera venta u otra transferencia de propiedad del original o de un ejemplar de la obra con autorización del autor. Declaración concertada respecto de los artículos 6 y 7: Tal como se utilizan en estos artículos, las expresiones "copias" y "originales y copias" sujetas al derecho de distribución y al derecho de alquiler en virtud de dichos artículos, se refieren exclusivamente a las copias fijadas que se pueden poner en circulación como objetos tangibles". Organización Mundial de la Propiedad Intelectual, OMPI, Tratado de la OMPI sobre Derecho de Autor de 1996 (WCT), Ginebra, 20 de diciembre de 1996, artículo 6 y declaración concertada. Disponible en: http://www.wipo.int/treaties/es/text.jsp?file_id=295167

9 Tueerd Overdijk, Polo van der Putt, Eva de Vries \& Thomas Schafft, Exhaustion and Software Resale Rights. A Comparison between the European Exhaustion Doctrine and the U.S. First Sale Doctrine in the Light of Recent Case Law, 12 Computer Law Review International, CRi, 2, 33-39 (2011). Disponible en: http://www.vondst-law.com/files/Exhaustion\%20and\%20 Software\%20Resale\%20Rights\%20CRI\%202011-02.pdf

10 Antoni Rubi-Puig, Copyright Exhaustion Rationales and Used Software: A Law and Economics Approach to Oracle v. UsedSoft, 4 Journal of Intellectual Property, Information Technology and e-Commerce Law, JIPITEC, 3, 159-178, 162 (2013). Disponible en: http://www.jipitec.eu/issues/ jipitec-4-3-2013/3842/rubi-puig.pdf. Yonatan Even, Appropriability, First Sale \& Exhaustion, 1-77, 5-10 (September 28, 2008). Disponible en SSRN: http://ssrn.com/abstract=1274822 
habilita a quien recibe esa copia a beneficiarse del agotamiento de los derechos de propiedad intelectual con el fin de poder revender este material ${ }^{11}$; hasta las discusiones propias de un mercado digital en el cual cada acto de distribución conlleva necesariamente una reproducción ${ }^{12}$, es posible calificar la figura del agotamiento como una institución en constante evolución ${ }^{13}$.

\section{A. La interpretación de los contratos de licencia}

Uno de los aspectos antes mencionados y que tiene una gran incidencia en la determinación del agotamiento de los derechos de propiedad intelectual, es la interpretación de los contratos mediante los cuales se entrega una copia de la obra protegida; en el caso de la industria del software, estos corresponden a los contratos de licencia. Si nos concentramos únicamente en la precisión hermenéutica empleada en la denominación de estos actos jurídicos, no cabría discusión alguna sobre la inoperatividad de la figura del agotamiento de los derechos de propiedad intelectual, a partir de una licencia de software, en la medida en que, como se dijo anteriormente, mediante una licencia no se transfieren derechos de propiedad intelectual, simplemente se autoriza su uso y/o goce; en palabras de la mayoría de las normas que regulan la materia, no habría una primera venta.

No obstante lo anterior, esta claridad no existe. Aun cuando los contratos de software son calificados como "licencias", se discute entre las partes si la entrega de la copia puede dar pie al agotamiento del derecho de distribución; y esta discusión se plantea desde la existencia o inexistencia de un acto dispositivo respecto de la copia entregada, es decir, si ocurre o no la "primera venta". Aunque no se puede desconocer la existencia de algunas posiciones que sostienen

11 Keith Harris, For Promotional Use Only: Is the Resale of a Promotional CD Protected by the First Sale Doctrine?, 30 Cardozo Law Review, 4, 1745-1772 (2009). Disponible en: http:// cardozolawreview.com/Joomla1.5/content/30-4/HARRIS.30-4.pdf

12 Lukas FeILER, Birth of the First-Download Doctrine: The Application of the First-Sale Doctrine to Internet Downloads under EU and U.S. Copyright Law, 1-18 (Transatlantic Technology Law Forum, TTLF, Working Paper 17, Stanford, Vienna, February 2013). Disponible en: https:// law.stanford.edu/publications/birth-of-the-first-download-doctrine-the-application-of-thefirst-sale-doctrine-to-internet-downloads-under-eu-and-u-s-copyright-law/

13 Más aún en el campo del software, Louise Longdin \& PHeH Hoon Lim, Inexhaustible Distribution Rights for Copyright Owners and the Foreclosure of Secondary Markets for Software, 44 International Review of Intellectual Property and Competition Law, 5, 541-568 (August 2013). 
que un contrato de licencia también otorga una licencia sobre el soporte material ${ }^{14}$ debido a la naturaleza del contrato mismo ${ }^{15}$, se puede afirmar que las condiciones bajo las cuales se entrega esa copia material en la mayoría de los casos han de ser interpretadas desde el acuerdo para poder determinar si ocurrió ese acto dispositivo en consideración a la falta de pacto contractual.

La interpretación tradicional de los contratos en los sistemas de derecho civil ${ }^{16}$, herederos del "voluntarismo", ha instrumentalizado esta función con el propósito, casi exclusivo, de "desentrañar el verdadero sentido de los actos jurídicos"17 cuando la voluntad de las partes no aparezca con toda claridad en el mismo texto ${ }^{18}$. Visión opuesta a la utilizada por el sistema objetivo de la interpretación de los contratos, para el cual la interpretación no tiene como finalidad encontrar las íntimas intenciones de las partes involucradas, buscando, en cambio, cómo los términos utilizados en el contrato pudieron haber sido razonable u objetivamente entendidos por los contratantes $^{19}$. Este último sistema es el que ha caracterizado la teoría más ortodoxa de los sistemas jurídicos del common law; de allí que el acercamiento objetivo a la interpretación de los contratos en esta tradición jurídica cada vez sufra más presiones evolutivas por parte de criterios adicionales que se distancian de una pura

14 Christina Bohannan \& Herbert Hovenkamp, Creation without Restraint: Promoting Liberty and Rivalry in Innovation, 371 (Oxford University Press, New York, 2012).

15 Orit Fischman-Afori, Implied License - An Emerging New Standard in Copyright Law, 25 Santa Clara High Technology Law Journal, 2, 275-325 (2009). Disponible en: http://digitalcommons. law.scu.edu/cgi/viewcontent.cgi?article $=1470 \&$ context $=$ chtlj

16 Denominada interpretación subjetiva, plenamente aplicable en países como Colombia y Chile. Jorge López SANTA-María, Algunos aspectos de la interpretación de los contratos en Chile, en Tratado de la interpretación del contrato en América Latina: Reglas para la interpretación de los contratos en América Latina, Tres tomos, 1215-1246 (CARlos Alberto Soto-Coaguila, dir, Editora Jurídica Grijley, Lima, 2007).

17 Colombia, Corte Suprema de Justicia, Sentencia del 27 de agosto de 1971, magistrado ponente Ernesto Cediel-Ángel.

18 Carlos Ignacio Jaramillo-Jaramillo, La interpretación del contrato en el derecho privado colombiano. Panorámico examen legal, jurisprudencial y doctrinal, en Tratado de la interpretación del contrato en América Latina: Reglas para la interpretación de los contratos en América Latina, Tres tomos, 804-1021 (CARlos Alberto Soto-Coaguila, dir, Editora Jurídica Grijley, Lima, 2007).

19 Stephen A. Smith, Contract Theory (Oxford University Press, Oxford, 2007). 
hermenéutica contractual ${ }^{20}$, como el contexto económico, la seguridad jurídica, la eficiencia en los negocios, incluso, la buena $\mathrm{fe}^{21}$.

Casos como MAI Systems Corp. v. Peak Computer Inc. ${ }^{22}$, Microsoft Corp. v. Harmony Computers \& Electronics, Inc. ${ }^{23}$, en los cuales la interpretación formalista determinó el efecto de la licencia ${ }^{24}$, se enfrentan a decisiones como las de Vernor v. Autodesk, Inc. ${ }^{25}$, o Microsoft Corp. v. DAK Industries ${ }^{26}$, en los que se han considerado otros factores más allá del formalismo hermenéutico para concluir si un contrato de licencia tiene como efecto o no, la primera venta de la copia entregada al usuario final.

En el caso de los sistemas civilistas en los cuales la interpretación parece tener muy pocas variaciones a las reglas de los códigos civiles, la reciente inclusión del paradigma proteccionista del consumidor parece ser la única variable a las reglas tradicionales de la interpretación de contratos, pues la interpretación a favor de

20 Brian W. CARver, Why License Agreements Do Not Control Copy Ownership: First Sales and Essential Copies, 25 Berkeley Technology Law Journal, 1887-1954 (2010). Disponible en: http:// ssrn.com/abstract $=1586580$

21 En los sistemas de interpretación subjetiva no se evidencian presiones tan claras como en los sistemas objetivos, aunque las evoluciones del concepto de buena fe contractual, como el deber de coherencia o el principio de solidaridad, pueden encontrarse como propuestas de nuevos paradigmas para la interpretación de contratos. MARIANA BERnAL-FANDiÑo, El deber de coherencia en el derecho colombiano de los contratos (Vicerrectoría Académica, Facultad de Ciencias Jurídicas, Editorial Pontificia Universidad Javeriana, Bogotá, 2013).

22 United States Court of Appeals for the Ninth Circuit, MAI Systems Corp. v. Peak Computer, Inc., 991 F.2d 511 (9 ${ }^{\text {th }}$ Cir. 1993), April 9, 1993. Disponible en: https://www.law.cornell.edu/ copyright/cases/991_F2d_511.htm

23 United States District Court for the Eastern District of New York, Microsoft Corp. v. Harmony Computers \& Electronics, Inc., 846 F. Supp. 208, February 11, 1994. Disponible en: http://law. justia.com/cases/federal/district-courts/FSupp/846/208/1687655/. United States District Court for the Eastern District of New York, Microsoft Corp. v. Harmony Computers \& Electronics, Inc., 846 F. Supp. 208, 31 U.S.P.Q.2d 1135, Copy. L. Rep. (CCH) P27,257, February 7, 1994.

24 La Corte del Noveno Circuito simplemente verificó la inclusión de la palabra licencia para determinar que los licenciatarios no podían alegar "la primera venta".

25 United States District Court for the Western District of Washington, Vernor v. Autodesk, Inc., 555 F. Supp. 2d 1164 (W. D. Wash. 2008), May 20, 2008.

26 United States Court of Appeals for the Ninth Circuit, Microsoft Corp. v. DAK Industries, 66 F.3d 1091 U.S. Dist (1995), October 2, 1995. Disponible en: http://openjurist.org/66/f3d/1091/ dak-industries-incorporated-microsoft-corporation-v-dak-industries-incorporated. En estas sentencias, el mismo Noveno Circuito introduce otros factores como las restricciones que el licenciante debe imponer al licenciatario para interpretar la existencia de la primera venta, como (i) que el licenciatario haya realizado una sola operación, no periodicidad; (ii) que el valor del contrato sea calculado de acuerdo al número de copias obtenidas; (iii) que el licenciatario reciba todos los derechos desde la vigencia del contrato; (iv) escenarios o casos en que el licenciatario debe devolver las copias entregadas por el licenciante. 
la parte débil parece ser el factor solitario para completar estos vacíos contractuales ${ }^{27}$.

En todo caso, las diferentes vertientes de las reglas de interpretación de contratos aparecerán solamente cuando el licenciatario considere que en virtud de la licencia se generó una primera disposición de la copia entregada o descargada, porque como se indicó anteriormente, las partes prestarán muy poca atención a la regulación contractual relacionada con esa copia y se concentrarán en las disposiciones tendientes a regular la licencia de los derechos de propiedad intelectual.

\section{B. El objeto sobre el que recae el agotamiento}

Sumado al problema de la interpretación de los contratos de licencia, las copias entregadas en virtud de las licencias de software no suelen ser fijaciones en soportes materiales, de forma que, de acuerdo a la declaración concertada respecto de los artículos 6 y 7 del Tratado de la OMPI sobre Derecho de Autor de 1996, hoy, en relación con un programa de computador objeto de descarga en internet no podría operar el agotamiento del derecho de distribución, en la medida en que esa descarga no es una "copia tangible", a diferencia de un $\mathrm{CD}$ u otro soporte material.

Este es el otro problema al que quiero referirme, y tal vez el más sensible de los que presento, toda vez que no importará si eventualmente se logra dar respuestas afirmativas a las demás preguntas planteadas en el punto II de esta presentación; si se entiende que el agotamiento únicamente puede operar respecto de copias materiales $^{28}$, en la práctica se afirma que hoy la figura del agotamiento es inaplicable para la mayoría de obras protegidas y muy especialmente respecto de los programas de computador ${ }^{29}$.

27 “Artículo 34. Interpretación favorable: Las condiciones generales de los contratos serán interpretadas de la manera más favorable al consumidor. En caso de duda, prevalecerán las cláusulas más favorables al consumidor sobre aquellas que no lo sean”. Colombia, Ley 1480 de 2011, por medio de la cual se expide el Estatuto del Consumidor y se dictan otras disposiciones, 48.220 Diario Oficial, 12 de octubre de 2011, artículo 34. Disponible en: http://www. secretariasenado.gov.co/senado/basedoc/ley_1480_2011.html

28 Sapna Kumar, Regulating Digital Trade, 67 Florida Law Review, 6, 1909-1959 (2015). Disponible en: http://www.floridalawreview.com/wp-content/uploads/7-Kumar.pdf

29 LuKas FeILER, Birth of the First-Download Doctrine: The Application of the First-Sale Doctrine to Internet Downloads under EU and U.S. Copyright Law, 1-18 (Transatlantic Technology Law Forum, TTLF, Working Paper 17, Stanford, Vienna, February 2013). Disponible en: https:// 
Esta misma línea de pensamiento es sostenida en relación con las obras musicales digitales en la sentencia Capitol Records, LLC v. ReDigi Inc. de $2013^{30}$, la cual reafirma que la defensa del agotamiento del derecho de distribución está limitada a bienes materiales; en este caso, reconoce además la imposibilidad de revender la misma copia digital que ha sido descargada originalmente por el comprador $^{31}$.

Como contrapunto, esta posición se confronta con la sentencia UsedSoft GmbH vs. Oracle International Corp., del 3 de julio de 2012, del Tribunal de Justicia de la Unión Europea. En ese caso, el alto Tribunal afirmó que el agotamiento del derecho de distribución operó aun cuando contractualmente se haya dispuesto que el software se entregaba en virtud de una licencia; se argumentó que "la descarga en el servidor del cliente de la copia del programa de ordenador que se encuentra en la página web del titular de los derechos y la celebración de un contrato de licencia de uso relativo a esa copia forman un todo indivisible que debe clasificarse, en su conjunto, de venta". Es decir, se verificó la primera venta aun cuando el origen haya sido un contrato de licencia.

Aunque en la sentencia UsedSoft el Tribunal de Justicia de la Unión Europea realizó un ejercicio de interpretación sobre el contrato de licencia, en el cual no se acordó explícitamente el título bajo el cual se entregaba la copia, para concluir que la licencia de uso y la descarga conforman en un todo una "primera venta" para efectos de aplicar el agotamiento de los derechos de propiedad intelectual, lo cierto es que esta interpretación no incluyó reglas o principios bajo los cuales un acto jurídico pueda ser interpretado como una disposición de la copia del software (como sí aparece en la sentencia Microsoft Corp. v. DAK Industries) ${ }^{32}$, sino que por

law.stanford.edu/publications/birth-of-the-first-download-doctrine-the-application-of-thefirst-sale-doctrine-to-internet-downloads-under-eu-and-u-s-copyright-law/

30 United States District Court for the Southern District of New York, Capitol Records, LLCv. ReDigi Inc., No. 12-0095, 2012 U.S. Dist (2013), March 30, 2013. Disponible en: http://digitalcommons. law.scu.edu/cgi/viewcontent.cgi?article $=1334 \&$ context $=$ historical

31 Shubra Grosh, The Implementation of Exhaustion Policies: Lessons from National Experiences, 1-85 (University of Wisconsin Law School, Legal Studies Research Paper Series Paper 1248, 2014). Disponible en: http://ssrn.com/abstract=2390232, http://www.ictsd.org/ downloads/2014/01/the-implementation-of-exhaustion-policies.pdf

32 United States Court of Appeals for the Ninth Circuit, Microsoft Corp. v. DAK Industries, 66 F.3d 1091 U.S. Dist (1995), October 2, 1995. Disponible en: http://openjurist.org/66/f3d/1091/ dak-industries-incorporated-microsoft-corporation-v-dak-industries-incorporated 
el contrario, recurrió a la finalidad garantista que propugna por proteger al consumidor, al aislar tal propósito de cualquier otro factor como la sostenibilidad de sus decisiones en el mercado.

No obstante, el caso UsedSoft constituye uno de los mejores ejemplos de la interpretación de contratos a favor del consumidor, carece de argumentos de peso para rebatir la inaplicabilidad del agotamiento a las copias digitales de un programa de computador, toda vez que esa sentencia centra toda su argumentación en la condición de lex specialis de la Directiva 2009/24 frente a la Directiva 2001/29/CE ${ }^{33}$, para concluir que al no haberse regulado una condición en ese sentido en la última Directiva, esta no puede ser un requisito de viabilidad para que opere el agotamiento en el caso de los programas de computador.

\section{Los bienes digitales tangibles}

El problema del tipo de copias sobre las cuales puede recaer el agotamiento del derecho de distribución debe ser analizado más allá de la simple consagración normativa. Como se ha visto, en la Directiva Europea 2009/24, como en otras legislaciones ${ }^{34}$, no se hace referencia a la limitación de la copia material; sin embargo, sí existe una norma de carácter internacional que suele ser presentada como el germen de esta limitación. Por eso resulta indispensable preguntarse por el alcance de esta disposición del Tratado OMPI de $1996^{35}$ :

33 Unión Europea, Directiva Europea 2009/24 del Parlamento Europeo y del Consejo, de 23 de abril de 2009, sobre la protección jurídica de los programas de ordenador. Disponible en: http:// eur-lex.europa.eu/legal-content/ES/TXT/?uri=uriserv\%3Ami0016. Unión Europea, Directiva Europea 2001/29/CE del Parlamento Europeo y del Consejo, de 22 de mayo de 2001, relativa a la armonización de determinados aspectos de los derechos de autor y derechos afines a los derechos de autor en la sociedad de la información. Disponible en: http://eur-lex.europa.eu/ legal-content/ES/TXT/?uri=CELEX\%3A32001L0029

34 Por ejemplo, la chilena (Ley 17.336) no hace referencia el tipo de copia; o la colombiana (Ley 23 de 1982 / Decisión Andina 351) no hace referencia al agotamiento en general. LuIs ÁngeL MADRID, Importaciones paralelas. Agotamiento del derecho de propiedad intelectual (Universidad Sergio Arboleda, octubre de 2005). Disponible en: http://190.85.246.40/tlc/tlc_importaciones \%20paralelas.htm. Chile, Ley 17.336 sobre la Propiedad Intelectual (modificada por la Ley 20.435, 4 de mayo de 2010), 28 de agosto de 1970, en vigor desde el 2 de octubre de 1970. Disponible en: http://www.wipo.int/wipolex/es/details.jsp?id=797. Colombia, Ley 23 de 1982, sobre derechos de autor, 35.949 Diario Oficial, 19 de febrero de 1982. Disponible en: http://www. alcaldiabogota.gov.co/sisjur/normas/Normal.jsp?i=3431. Comisión del Acuerdo de Cartagena, Decisión Andina 351, Régimen Común sobre Derecho de Autor y Derechos Conexos, 17 de diciembre de 1993. Disponible en: http://www.wipo.int/wipolex/es/text.jsp?file_id=223497

35 Además de otras normas supranacionales o nacionales como la Directiva Europea 2001/29/CE. 
Artículo 6. Derecho de distribución:

(1) Los autores de obras literarias y artísticas gozarán del derecho exclusivo de autorizar la puesta a disposición del público del original y de los ejemplares de sus obras mediante venta u otra transferencia de propiedad.

(2) Nada en el presente Tratado afectará la facultad de las partes contratantes de determinar las condiciones, si las hubiera, en las que se aplicará el agotamiento del derecho del párrafo 1) después de la primera venta u otra transferencia de propiedad del original o de un ejemplar de la obra con autorización del autor.

Declaración concertada respecto de los artículos 6 y 7: Tal como se utilizan en estos artículos, las expresiones "copias" y "originales y copias" sujetas al derecho de distribución y al derecho de alquiler en virtud de dichos artículos, se refieren exclusivamente a las copias fijadas que se pueden poner en circulación como objetos tangibles.

En mi opinión, hoy resulta imposible identificar de forma exclusiva el concepto de "copias tangibles" con el de "copias materiales". Partiendo del principio de equivalencia funcional establecido el artículo 5 de la Ley Modelo de la CNUDMI sobre Comercio Electrónico y adoptado en Colombia por la Ley 527 de $1999^{36}$, se encuentra la manifestación de la necesidad social de equiparar los efectos de los soportes (bienes) digitales con los de aquellos soportes materiales o analógicos; hasta llegar al más tradicional principio de distinción entre el corpus mysticum y el corpus mechanicum, el concepto de "copia tangible" debe ampliar su espectro más allá de las "copias materiales" 37 .

Entonces, propongo entender la declaración concertada del artículo 6 del Tratado OMPI como una reafirmación de la imposibilidad de que el agotamiento opere sobre bienes inmateriales, como el derecho de distribución en abstracto, el cual nunca se agotaría

36 Colombia, Corte Constitucional, Sentencia C-662-00, 8 de junio de 2000, magistrado ponente Fabio Morón Díaz. Disponible en: http://www.corteconstitucional.gov.co/ relatoria/2000/C-662-00.htm

37 Para ver posiciones que sí equiparan estos dos conceptos: United States District Court for the Southern District of New York, Capitol Records, LLC v. ReDigi Inc., No. 12-0095, 2012 U.S. Dist (2013), March 30, 2013. Disponible en: http://digitalcommons.law.scu.edu/cgi/viewcontent. cgi?article $=1334 \&$ context $=$ historical. YeCID ANDRÉs Ríos-PInZón, El agotamiento del derecho de autor y los derechos conexos (contenido, alcance y aplicación a las transmisiones en línea), 1-51 (Centro Colombiano del Derecho de Autor, CECOLDA). Disponible en: http://www.cecolda. org.co/index.php/informacion/columnista-invitado/262-el-agotamiento-delderecho-de-autory-los-derechos-conexos-contenido-alcance-y-aplicacion-alas-transmisiones-en-linea 
en relación con la primera venta de una copia. Y afirmar, de esta manera, que el derecho de distribución en particular, referido a una copia, sí es susceptible de ser agotado aun cuando esa copia sea digital. La condición digital no disminuye la característica tangible de una copia, al fin y al cabo esta será determinada, tanto en su extensión como en su ubicación y, en consecuencia, también será singularizada; es decir, se podrá diferenciar de otras copias que puedan tener características similares. De allí el término "bienes digitales tangibles", mediante el cual, no solo se asegurará la aplicación de las mismas prerrogativas de los bienes analógicos en favor de aquellos que son digitales, sino que además se estará reconociendo una realidad social y jurídica innegable, la de la equivalencia funcional entre soportes.

Esta idea, aunque no muy extendida ${ }^{38}$, puede encontrarse en la sentencia Microsoft Corp. v. AT\&T Corp. ${ }^{39}$, en la cual la Corte Suprema de Estados Unidos ante una demanda de infracción de las patentes de AT\&T por parte de Microsoft, diferenció entre "información digital abstracta", la cual no constituye un componente violatorio de una patente (en este caso, en referencia al código fuente de un software) y la "información digital fijada" o "información digital en concreto", la cual sí sería catalogada como un componente, sin importar si esta última se manifiesta en soportes analógicos como un $\mathrm{CD}$, o en soportes digitales como una descarga de internet ${ }^{40}$.

38 Andreas Rahmatian, Intellectual Property and the Concept of Dematerialised Property, en Modern Studies in Property Law, Vol. 6, 361-383 (Susan Bright, ed., Hart Publishing, Oxford, 2011). Disponible en: http://ssrn.com/abstract $=1917950$

39 United States Supreme Court, Microsoft Corp. v. AT\&T Corp., 550 U.S. 437 (2007), April 30, 2007. Disponible en: https://supreme.justia.com/cases/federal/us/550/437/, http://www.supremecourt.gov/opinions/06pdf/05-1056.pdf

40 Sapna Kumar, Regulating Digital Trade, 67 Florida Law Review, 6, 1909-1959 (2015). Disponible en: http://www.floridalawreview.com/wp-content/uploads/7-Kumar.pdf 


\section{CONCLUSIONES}

La sentencia en el caso UsedSoft $t^{41}$ ha traído consigo innumerables reacciones desde todos los sectores que han llevado a calificarla favorablemente o de forma negativa con diferentes consideraciones jurídicas o de conveniencia social y económica; sin embargo, esta sentencia ha provocado un estudio que faltaba en las jurisdicciones de tradición civilista y que sí había sido muy estudiado en Estados Unidos, esto es la interpretación de los contratos de licencia de software.

Emprender este recorrido de interpretar los contratos de licencia de programas de computador podría significar una evolución de las reglas generales sobre la interpretación de los contratos que están tan arraigadas y son estables en los países de tradición civilista, en donde el esquivo y abnegado propósito de encontrar la verdadera intención de las partes, que en la actualidad manifiestan intereses contradictorios, está descartando otros objetivos igualmente importantes como la seguridad jurídica, los límites a la propiedad, la realidad económica o la estabilidad de un mercado ${ }^{42}$. Por su parte, el cambio de paradigma hacia un garantismo de la posición de los consumidores, aunque constituye una cierta actualización de los sistemas tradicionales de interpretación de los contratos, en la medida en que sea considerado como un elemento aislado, como lo ha venido siendo, derivará nuevamente en la negación de los objetivos olvidados por el voluntarismo tradicional.

Adicionalmente, encontramos que revitalizar el principio de distinción entre el corpus mysticum y el corpus mechanicum implica ponerse a tono con la equivalencia funcional entre soportes digitales y analógicos; más aún, en el caso concreto de la figura del agotamiento del derecho de distribución, su aplicación no puede quedar circunscrita a las copias analógicas, ya en desuso, y debe extenderse sin restricciones a todas las copias digitales, entendiendo este tipo de soporte bajo el concepto más amplio de bien digital tangible.

41 Tribunal de Justicia de la Unión Europea, TJUE, UsedSoft GmbH vs. Oracle International Corp., 3 de julio de 2012. Disponible en: http://curia.europa.eu/juris/document/document. jsf?docid $=124564 \&$ doclang $=E S$

42 Ariel Katz, What Antitrust Law Can (and Cannot) Teach about the First Sale Doctrine, 1-58, 6 (University of Toronto, Faculty of Law, Working paper, January 23, 2012). Disponible en: http://ssrn.com/abstract $=1845842$ 


\section{BIBLIOGRAFÍA}

\section{Libros}

Baylos-Corroza, Hermenegildo, Tratado de derecho industrial ( $3^{\mathrm{a}}$ ed., Civitas, Navarra, 2009).

Bernal-Fandiño, Mariana, El deber de coherencia en el derecho colombiano de los contratos (Vicerrectoría Académica, Facultad de Ciencias Jurídicas, Editorial Pontificia Universidad Javeriana, Bogotá, 2013).

Bohannan, Christina \& Hovenkamp, Herbert, Creation without Restraint: Promoting Liberty and Rivalry in Innovation (Oxford University Press, New York, 2012).

Smith, Stephen A., Contract Theory (Oxford University Press, Oxford, 2007).

\section{Colaboración en obras colectivas}

Jaramillo-Jaramillo, Carlos Ignacio, La interpretación del contrato en el derecho privado colombiano. Panorámico examen legal, jurisprudencial y doctrinal, en Tratado de la interpretación del contrato en América Latina: Reglas para la interpretación de los contratos en América Latina, Tres tomos, 804-1021 (CARLOS Alberto Soto-Coaguila, dir, Editora Jurídica Grijley, Lima, 2007).

López Santa-María, Jorge, Algunos aspectos de la interpretación de los contratos en Chile, en Tratado de la interpretación del contrato en América Latina: Reglas para la interpretación de los contratos en América Latina, Tres tomos, 1215-1246 (Carlos Alberto Soto-Coaguila, dir, Editora Jurídica Grijley, Lima, 2007).

Rahmatian, Andreas, Intellectual Property and the Concept of Dematerialised Property, en Modern Studies in Property Law, Vol. 6, 361-383 (Susan Bright, ed., Hart Publishing, Oxford, 2011). Disponible en: http://ssrn.com/abstract=1917950

\section{Revistas}

Carver, Brian W., Why License Agreements Do Not Control Copy Ownership: First Sales and Essential Copies, 25 Berkeley Technology Law Journal, 1887-1954 (2010). Disponible en: http://ssrn.com/abstract $=1586580$

Contreras-Jaramillo, Juan Camilo, Origen y sustento del agotamiento de los derechos de propiedad intelectual, 131 Vniversitas, 277-322 (2015). Disponible en: http:// revistas.javeriana.edu.co/index.php/vnijuri/article/view/15030/12399

Fischman-Afori, Orit, Implied License - An Emerging New Standard in Copyright Law, 25 Santa Clara High Technology Law Journal, 2, 275-325 (2009). Disponible en: http:// digitalcommons.law.scu.edu/cgi/viewcontent.cgi?article $=1470 \&$ context $=$ chtlj

Harris, Keith, For Promotional Use Only: Is the Resale of a Promotional CD Protected by the First Sale Doctrine?, 30 Cardozo Law Review, 4, 1745-1772 (2009). Disponible en: http://cardozolawreview.com/Joomla1.5/content/30-4/HARRIS.30-4.pdf 
Kumar, Sapna, Regulating Digital Trade, 67 Florida Law Review, 6, 1909-1959 (2015). Disponible en: http://www.floridalawreview.com/wp-content/uploads/7-Kumar. pdf

Longdin, Louise \& Lim, Phen Hoon, Inexhaustible Distribution Rights for Copyright Owners and the Foreclosure of Secondary Markets for Software, 44 International Review of Intellectual Property and Competition Law, 5, 541-568 (August 2013).

Overdijk, Tueerd; Putt, Polo van der; Vries, Eva de \& Schafft, Thomas, Exhaustion and Software Resale Rights. A Comparison between the European Exhaustion Doctrine and the U.S. First Sale Doctrine in the Light of Recent Case Law, 12 Computer Law Review International, CRi, 2, 33-39 (2011). Disponible en: http:// www.vondst-law.com/files/Exhaustion $\% 20$ and $\% 20$ Software $\% 20$ Resale $\% 20$ Rights\%20CRI\%202011-02.pdf

Rubí-Puig, Antoni, Copyright Exhaustion Rationales and Used Software: A Law and Economics Approach to Oracle v. UsedSoft, 4 Journal of Intellectual Property, Information Technology and e-Commerce Law, JIPITEC, 3, 159-178 (2013). Disponible en: http://www.jipitec.eu/issues/jipitec-4-3-2013/3842/rubi-puig.pdf

Westкаmp, Guido, Intellectual Property, Competition Rules and the Emerging Internal Market: Some Thoughts on the European Exhaustion Doctrine, 11 Marquette Intellectual Property Law Review, 2, 291-335 (2007). Disponible en: http:// scholarship.law.marquette.edu/cgi/viewcontent.cgi?article=1096\&context=iplr

\section{Working paper, artículo de opinión}

Contreras-Jaramillo, JuAn CAmilo, El aparato teórico en la estructura tradicional del sistema de propiedad intelectual (Pontificia Universidad Javeriana, Working paper, enero de 2016). Disponible en: https://www.academia.edu/25275568/ WORKING_PAPER_El_Aparato_Te\%C3\%B3rico_en_la_Estructura_ Tradicional_del_Sistema_de_Propiedad_Intelectual

Even, Yonatan, Appropriability, First Sale \& Exhaustion (September 28, 2008). Disponible en SSRN: http://ssrn.com/abstract $=1274822$

Feiler, Lukas, Birth of the First-Download Doctrine: The Application of the FirstSale Doctrine to Internet Downloads under EU and U.S. Copyright Law, 1-18 (Transatlantic Technology Law Forum, TTLF, Working Paper 17, Stanford, Vienna, February 2013). Disponible en: https://law.stanford.edu/publications/ birth-of-the-first-download-doctrine-the-application-of-the-first-sale-doctrineto-internet-downloads-under-eu-and-u-s-copyright-law/

Grosh, Shubha, The Implementation of Exhaustion Policies: Lessons from National Experiences, 1-85 (University of Wisconsin Law School, Legal Studies Research Paper Series Paper 1248, 2014). Disponible en: http://ssrn.com/abstract=2390232, http://www.ictsd.org/downloads/2014/01/the-implementation-of-exhaustionpolicies.pdf

Katz, Ariel, What Antitrust Law Can (and Cannot) Teach about the First Sale Doctrine, 1-58 (University of Toronto, Faculty of Law, Working paper, January 23, 2012). Disponible en: http://ssrn.com/abstract $=1845842$

MAdrid, Luis Ángel, Importaciones paralelas. Agotamiento del derecho de propiedad 
intelectual (Universidad Sergio Arboleda, octubre de 2005). Disponible en: http://190.85.246.40/tlc/tlc_importaciones\%20paralelas.htm

Ríos-Pinzón, YeCid AndRés, El agotamiento del derecho de autor y los derechos conexos (contenido, alcance y aplicación a las transmisiones en línea), 1-51 (Centro Colombiano del Derecho de Autor, CECOLDA). Disponible en: http://www. cecolda.org.co/index.php/informacion/columnista-invitado/262-el-agotamientodelderecho-de-autor-y-los-derechos-conexos-contenido-alcance-y-aplicacionalas-transmisiones-en-linea

\section{Tratados internacionales}

Organización Mundial de la Propiedad Intelectual, OMPI, Tratado de la OMPI sobre Derecho de Autor de 1996, Ginebra, 20 de diciembre de 1996. Disponible en: http://www.wipo.int/treaties/es/text.jsp?file_id=295167

\section{Normatividad internacional}

Chile, Código Civil, 14 de diciembre de 1855; Decreto con Fuerza de Ley 1 de 2000, 16 de mayo de 2000, fija texto refundido, coordinado y sistematizado del Codigo Civil; de la Ley 4.808, sobre registro civil; de la Ley 17.344, que autoriza cambio de nombres y apellidos; de la Ley 16.618, ley de menores; de la Ley 14.908, sobre abandono de familia y pago de pensiones alimenticias, y de la Ley 16.271, de impuesto a las herencias, asignaciones y donaciones. Disponible en: http://www. leychile.cl/Navegar?idNorma $=172986$

Chile, Ley 17.336 sobre la Propiedad Intelectual (modificada por la Ley 20.435, 4 de mayo de 2010), 28 de agosto de 1970, en vigor desde el 2 de octubre de 1970. Disponible en: http://www.wipo.int/wipolex/es/details.jsp?id=797

Comisión de las Naciones Unidas para el Derecho Mercantil Internacional, CNUDMI/ UNCITRAL, Ley Modelo de la CNUDMI sobre Comercio Electrónico, 12 de junio de 1996. Disponible en: http://www.uncitral.org/uncitral/es/uncitral_ texts/electronic_commerce.html, https://www.uncitral.org/pdf/spanish/texts/ electcom/05-89453_S_Ebook.pdf

Comisión del Acuerdo de Cartagena, Decisión Andina 351, Régimen Común sobre Derecho de Autor y Derechos Conexos, 17 de diciembre de 1993. Disponible en: http://www.wipo.int/wipolex/es/text.jsp?file_id=223497

Estados Unidos, Copyright Act of 1976, 17 United States Code $\S 109$. Disponible en: http://www.copyright.gov/title17/92chap1.html

Unión Europea, Directiva Europea 2001/29/CE del Parlamento Europeo y del Consejo, de 22 de mayo de 2001, relativa a la armonización de determinados aspectos de los derechos de autor y derechos afines a los derechos de autor en la sociedad de la información. Disponible en: http://eur-lex.europa.eu/legal-content/ES/ TXT/?uri=CELEX\%3A32001L0029

Unión Europea, Directiva Europea 2009/24 del Parlamento Europeo y del Consejo, de 23 de abril de 2009, sobre la protección jurídica de los programas de 
ordenador. Disponible en: http://eur-lex.europa.eu/legal-content/ES/ TXT/?uri=uriserv $\% 3 \mathrm{Ami} 0016$

\section{Normatividad colombiana}

Colombia, Dirección de Impuestos y Aduanas Nacionales, DIAN, Concepto 063288, 16 de agosto de 2007. Disponible en: http://www.notinet.com.co/pedidos/conc63288-07.doc

Colombia, Ley 57 de 1887, Código Civil. Disponible en: http://www.secretariasenado. gov.co/senado/basedoc/codigo_civil.html

Colombia, Ley 23 de 1982, sobre derechos de autor, 35.949 Diario Oficial, 19 de febrero de 1982. Disponible en: http://www.alcaldiabogota.gov.co/sisjur/normas/Norma1. jsp?i=3431

Colombia, Ley 1480 de 2011, por medio de la cual se expide el Estatuto del Consumidor y se dictan otras disposiciones, 48.220 Diario Oficial, 12 de octubre de 2011. Disponible en: http://www.secretariasenado.gov.co/senado/basedoc/ ley_1480_2011.html

Colombia, Ley 527 de 1999, por medio de la cual se define y reglamenta el acceso y uso de los mensajes de datos, del comercio electrónico y de las firmas digitales, y se establecen las entidades de certificación y se dictan otras disposiciones, 43.673 Diario Oficial, 21 de agosto de 1999. Disponible en: http://www.secretariasenado. gov.co/senado/basedoc/ley_0527_1999.html

\section{Casos}

Tribunal de Justicia de la Unión Europea, TJUE, UsedSoft GmbH vs. Oracle International Corp., 3 de julio de 2012. Disponible en: http://curia.europa.eu/juris/document/ document.jsf?docid $=124564 \&$ doclang $=\mathrm{ES}$

United States Court of Appeals for the Ninth Circuit, MAI Systems Corp. v. Peak Computer, Inc., 991 F.2d 511 (9 $9^{\text {th }}$ Cir. 1993), April 9, 1993. Disponible en: https:// www.law.cornell.edu/copyright/cases/991_F2d_511.htm

United States Court of Appeals for the Ninth Circuit, Microsoft Corp. v. DAK Industries, 66 F.3d 1091 U.S. Dist (1995), October 2, 1995. Disponible en: http://openjurist. org/66/f3d/1091/dak-industries-incorporated-microsoft-corporation-v-dakindustries-incorporated

United States District Court for the Eastern District of New York, Microsoft Corp. v. Harmony Computers \& Electronics, Inc., 846 F. Supp. 208, February 11, 1994. Disponible en: http://law.justia.com/cases/federal/district-courts/ FSupp/846/208/1687655/

United States District Court for the Eastern District of New York, Microsoft Corp. v. Harmony Computers \& Electronics, Inc., 846 F. Supp. 208, 31 U.S.P.Q.2d 1135, Copy. L. Rep. (CCH) P27,257, February 7, 1994.

United States District Court for the Southern District of New York, Capitol Records, 
LLC v. ReDigi Inc., No. 12-0095, 2012 U.S. Dist (2013), March 30, 2013. Disponible en: http://digitalcommons.law.scu.edu/cgi/viewcontent.cgi?article $=1334 \&$ conte $\mathrm{xt}=$ historical

United States District Court for the Western District of Washington, Vernor v. Autodesk, Inc., 555 F. Supp. 2d 1164 (W. D. Wash. 2008), May 20, 2008.

United States Supreme Court, Microsoft Corp. v. AT\&T Corp., 550 U.S. 437 (2007), April 30, 2007. Disponible en: https://supreme.justia.com/cases/federal/us/550/437/, http://www.supremecourt.gov/opinions/06pdf/05-1056.pdf

\section{Jurisprudencia colombiana}

Colombia, Corte Constitucional, Sentencia C-662-00, 8 de junio de 2000, magistrado ponente Fabio Morón Díaz. Disponible en: http://www.corteconstitucional.gov. co/relatoria/2000/C-662-00.htm

Colombia, Corte Suprema de Justicia, Sentencia del 27 de agosto de 1971, magistrado ponente Ernesto Cediel-Ángel. 
